Case Report

\title{
VARICELLA ZOSTER VIRAL RETINITIS IN A PATIENT WITH SYSTEMIC LUPUS ERYTHEM ATOSIS
}

\author{
Kalpana Badami Nagaraj ${ }^{1}$, Sonali Muralidhar ${ }^{2}$, Chaitra Jayadev ${ }^{3} \&$ Kirthiraj $^{4}$ \\ ${ }^{1}$ Head of Vitreoretina Services, Minto Ophthalmic Hospital, Bangalore, \\ ${ }^{2,3,4}$ VR Fellow, Minto Ophthalmic Hospital, Bangalore. \\ Correspondence: \\ Badami Kalpana Nagaraj \\ Head of Vitreoretina Department, M into Ophthalmic Hospital, AV Road, Opp Central Police Station, Chamarajpet, \\ Bangalore 560002, Karnataka, India. \\ Mobile : +91 9448040627 E-mail : badamikal@gmail.com
}

\begin{abstract}
:
A young female a known case of systemic lupus erythematosus who underwent renal transplantation for end stage lupus nephritis was on long term immunosuppression for her systemic condition. She had an episode of varicella three months before she presented to us with ocular complaints in the right eye. She was diagnosed to have progressive outer retinal necrosis with a differential of cytomegaloviral retinitis in the right eye and treatment was initiated. She had begun to show improvement in visual acuity but within two weeks her systemic condition worsened rapidly and she succumbed to septic shock.

The ocular infection in an immunocompromised SLE patient can be a precursor of mortality as seen in our patient.

Due consideration is to be given for prophylactic treatment post varicella infection along with a multidisciplinary approach in managing the immunocompromised, to prevent devastating complications or mortality.
\end{abstract}

Keywords: Viral retinitis, immunosuppressants,systemic lupus

\section{Introduction:}

Systemic lupus erythematosis (SLE) is a chronic, autoimmune, multisystem disease which entails treatment with long term immunosuppressants. We report a case of varicella zoster viral (VZV) retinitis, three months after a primary infection of varicella in a seronegative SLE patient who was on immunosuppressants for a renal transplantation.

\section{Case report:}

In Feb 2014, a 16-year-old female with a history of systemic lupus erythematosus, presented with sudden, painless diminution of vision associated with floaters in the right eye of one-week duration.

Access this article online Quick Response Code

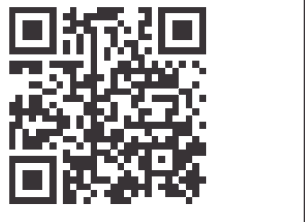

One and a half years before the onset of her ocular complaints, she had had an episode of arthralgia and oliguria for which she was hospitalized and diagnosed to have SLE that fulfilled the American College of Rheumatology criteria. Renal biopsy report had confirmed a Class 4 lupus nephritis. Treatment as per the Euro-Lupus protocol and maintenance haemodialysis had been rendered. A diagnosis of resistant lupus was subsequently made owing to failure of adequate response to immunosuppressants. Treatment with mycophenolate mofetil 500mg twice daily, prednisolone $20 \mathrm{mg}$ once daily, hydroxychloroquine $200 \mathrm{mg}$ twice daily was initiated. In October 2013 live related renal transplantation with $\mathrm{ABO}$ incompatibility was performed. Post transplantation triple immunosuppression was administered, tacrolimus $3.25 \mathrm{mg}$ once daily, mycophenolate mofetil $500 \mathrm{mg}$ twice daily and prednisolone $10 \mathrm{mg}$ once daily. In December 2013, characteristic vesicular lesions on the face, trunk, and extremities noted was diagnosed as varicella and conservatively managed. She had frequent follow-ups without any ocular complaints until M arch 2014 when she developed a sudden, painless, diminution of vision in the right eye and was referred to the ophthalmologist. A diagnosis of branch retinal vein occlusion was made 
elsewhere and referred to us.

In our centre, ocular examination revealed a best-corrected visual acuity of 20/100 in the right eye and of 20/20 in the left eye. Slit lamp examination of the right eye showed no aqueous cells or flare and no anterior or posterior vitreous cells. Fundus examination revealed deep retinal discolouration around the fovea with superficial haemorrhages along the superior arcade (Figure 1). Vasculitis was noted nasal to the disc. The peripheral fundus showed a featureless retina with retinal pigment epithelium stippling. The left eye examination was unremarkable. Due to the paucity of reaction and the deep retinal lesions a presumptive diagnosis of progressive outer retinal necrosis in the right eye was made. The presence of vasculitis was confounding due to which a differential diagnosis of acute retinal necrosis and cytomegalovirus retinitis (CM V) was considered. An aqueous sample was sent for polymerase chain reaction (PCR) analysis for cytomegalovirus and varicella-zoster virus. Nested PCR was positive for CMV. Real time PCR was positive for VZV and weakly positive for CM V. Other blood investigations revealed non reactive human immunodeficiency virus 1 and 2 , negative hepatitis- $B$ surface antigen and a negative venereal disease research laboratory test for syphilis. Her visual acuity rapidly deterio rated to perception of light with a normal projection of rays within two days. The deep retinal lesions which were initially fovea sparing were now seen to involve the fovea. Intravenous injection of gancyclovir $250 \mathrm{mg}$ twice daily was started and maintenance immunosuppresants were continued. Intravitreal ganciclovir $0.05 \mathrm{mg} / 0.1 \mathrm{ml}$ was given to her right eye. Five days later her visual acuity had marginally improved to counting fingers close to face with the fundus showing absorbing retinal haemorrhages and resolving retinitis patches (Figure 2). Subsequently oral valganciclovir 450 mg twice daily, dose corrected for her reduced creatinine clearance was administered. Her visual acuity was stable at the last follow up but without significant improvement. Subsequently her systemic condition worsened within two weeks requiring admission to the intensive care unit where she succumbed to septic shock.
Figure 1 : Right eye posterior pole at presentation

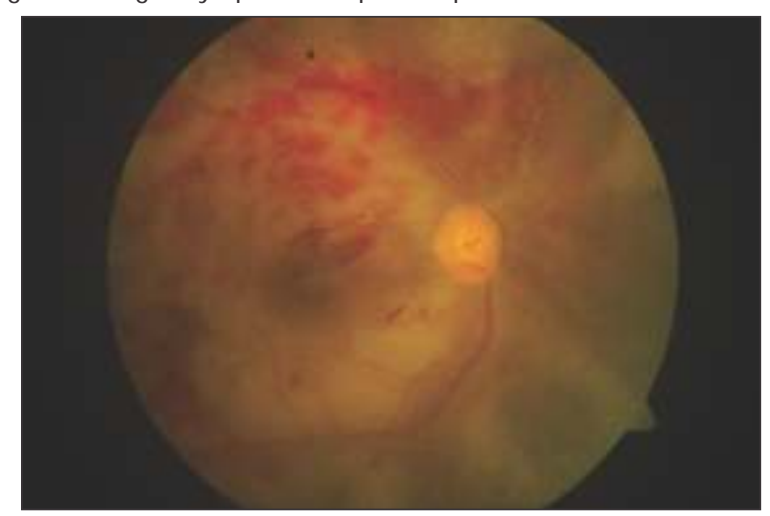

The posterior pole shows deep yellow retinal lesion inferior to fovea, measuring around 4 disc diameters in size with haemorrhages and creamy white necrotic retinitis patches along the superotemporal arcade.

Figure 2 : Right eye fundus post intravitreal injection

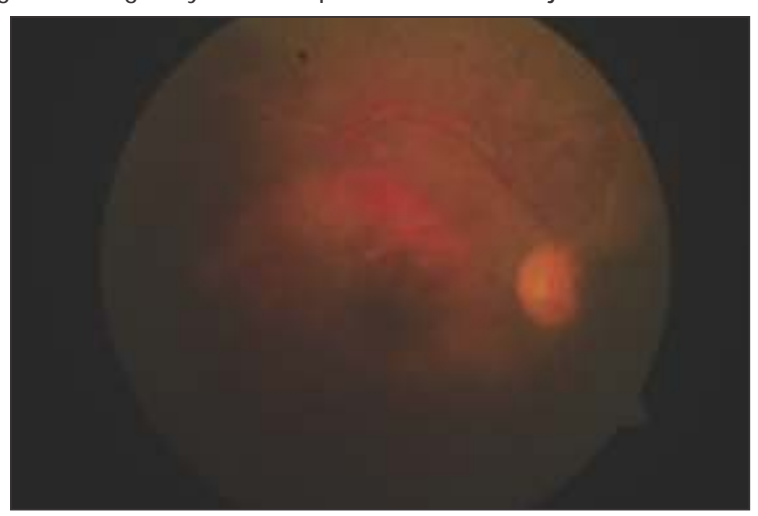

The posterior pole of the right eye after treatment shows absorbing retinal haemorrhages and resolving retinitis patches.

\section{Discussion:}

In SLE patients ocular involvement is seen in a third of patients due to several mechanisms including immune complex deposition, antibody related mechanisms, vasculitis and thrombosis. ${ }^{[1]}$

Rarely, retinal infections due to herpes simplex, varicella zoster and CM V can be seen in them. ${ }^{[2]}$

In SLE patients, VZV retinitis is less commonly reported. Matthews BN et al reported a case of unilateral varicella zoster virus ophthalmicus and contralateral acute retinal necrosis in an SLE patient. ${ }^{[3]}$ Our case of VZV retinitis, three months following varicella infection is unique with no previous episode of herpes zoster. 
Considering the fundus picture and the PCR reports there is a remote possibility of a combined infection of VZV and CM V in our patient.

Pavesio et al have suggested that intravitreal antivirals may be useful in the management of VZV retinitis since high levels of ganciclovir can be attained in the vitreous cavity and retina. ${ }^{[4]}$ Combined intravenous and intravitreal antiviral therapy may help control viral replication in the early active phases of the disease. ${ }^{[5]}$

In our patient, improvement was observed after the treatment with intravitreal gancyclovir and oral valganciclovir, despite the inability to reduce the dosage of the immunosuppressants due to her systemic disease.

\section{References:}

1. Sivaraj RR, Durrani OM, Denniston AK, Murray PI, Gordon C. Ocular manifestations of systemic lupus erythematosus. Rheumatology 2007; 46 :1757-1762.

2. Juneja R, Sune P, Kamble M, Subramanian P, Dulani, S, Sune M. An Unusual Case of Retinal Vasculitis and Neuroretinitis with Chronic Glomerulonephritis in Sytemic Lupus Erythematosus with Hughes Syndrome. The Internet Journal of Ophthalmology and Visual Science. 2012; 9(1): [about 2 pages]. Available from http://www. ispub.com/IJOVS/9/1/14338

3. Matthews B N , Erb N, Gordon C, Callear A B , M urray P I, Salmon M . Unilateral varicella zoster virus ophthalmicus and contralateral acute retinal necrosis. Eye 2002;16: 778-780.

4. Pavesio CE, Mitchell SM, Barton K, Schwartz S D, Towler H M A, Lightman S. Progressive outer retinal necrosis (PORN) in AIDS patients: a different appearance of varicella-zoster retinitis. Eye 1995; 9:271-6.

5. M oorthy R S, Weinberg D V, Teich SA, Berger B B, M inturn J T, Kumar S, et al. Management of varicella zoster virus retinitis in AIDS. Br J Ophthalmol 1997;81:189-194

\section{Conclusion:}

Progressive outer retinal necrosis syndrome secondary to VZV retinitis, has potentially devastating sight-threatening consequences with rapid progression despite early detection and intervention. There should be a high index of suspicion of infectious retinal necrosis, either due to CMV or VZV, in immunocompromised SLE patients post varicella infection.

The ocular infection in an immunocompromised SLE patient may precede mortality as seen in our patient.

Due consideration is to be given for prophylactic treatment post varicella infection along with a multidisciplinary approach in managing the immunocompromised, to prevent devastating complications or mortality. 